\title{
Facharztprüfungen in Rheumatologie: eine fortschrittliche Entwicklung über 10 Jahre
}

\author{
M. Weber ${ }^{a}$, R. Westkämper ${ }^{b}$, A. Aeschlimann', C. Beyeler ${ }^{\text {}}$
}

\author{
a Stadtspital Triemli, Zürich \\ b Institut für Medizinische Lehre, \\ Universität Bern \\ c RehaClinic, Zurzach \\ d Klinik und Polikliniken für \\ Rheumatologie und klinische \\ Immunologie/Allergologie, \\ Inselspital Bern
}

* Leserinnen und Leser sind gebeten, bei männlichen Formen auch die weibliche mitzudenken.

1 Salzberg R. Konzept Evaluation der Weiter- und Fortbildung. Schweiz Ärztezeitung 1990; 71:1298-309.

2 Westkämper R, Aeschlimann A. Facharztprüfung in der Rheumatologie. Schweiz Ärztezeitung 1996; 77:137-9.

3 Westkämper R, Aeschlimann A. Facharztprüfung in der Rheumatologie 1996. Schweiz Ärztezeitung 1997;78:660-3.

4 Beyeler C, Westkämper R, Villiger PM, Aeschlimann A. Selfassessment in continuous professional development: a valuable tool for individual physicians and scientific societies. Ann Rheum Dis 2004;63:1684-6.

5 Aeschlimann A, Westkämper R, Doherty M, Woolf AD. Multiplechoice question quiz: a valid test for needs assessments in CME in rheumatology and for self-assessment. Ann Rheum Dis 2001; 60:740-3.

6 Weber M. Facharztprüfung für Rheumatologie: «Vorbildlich, solid, objektiv». Rheuma Nachrichten 2001;25:3-5.

7 Weiterbildungsprogramm zum Facharzt für Rheumatologie. www.fmh.ch/shared/data/pdf/ rheumatologie_version_internet _d.pdf.

Korrespondenz:

Dr. med. Marcel Weber Stadtspital Triemli

Klinik für Rheumatologie und Rehabilitation

CH-8063 Zürich

E-Mail:

marcel.weber@triemli.stzh.ch

\section{Geschichtliche Einleitung}

Die obligatorischen Prüfungen zur Erlangung eines Facharzttitels und die nachweispflichtige kontinuierliche Fortbildung sind Ausdruck der Bemühungen, Standards festzusetzen und Qualitätsnormen zu überprüfen. Nach einer Urabstimmung durch die Schweizerische Ärzteschaft wurden diese Elemente der Qualitätssicherung in die Weiterbildungs- und Fortbildungsordnung aufgenommen und in der Folge schrittweise eingeführt [1].

Die Schweizerische Gesellschaft für Rheumatologie (SGR) hat die Facharztprüfungen konzipiert, um drei Ansprüchen gerecht zu werden. Erstens ermöglichen sie eine objektive und national einheitliche Beurteilung der Leistungen der Titelanwärter* als zukünftige Rheumatologen. Zweitens dienen sie den Fachgesellschaftsmitgliedern als Instrument der Selbsteinschätzung der eigenen Kompetenzen (Self-assessment). Drittens helfen sie den Verantwortlichen, den Bedarf an spezifisch zu vermittelnden Weiter- und Fortbildungsinhalten abzuschätzen (Needs-assessment). Um die Fachkompetenzen breit zu überprüfen, wurden in der Prüfungsordnung eine schriftliche Prüfung zur Beurteilung des Fachwissens und eine praktisch-mündliche Prüfung zur Erfassung der Anwendung der Kenntnisse, Fertigkeiten und Einstellungen vorgesehen.

Im Jahre 1995 wurde für die Teilnehmer eines SGR-Fortbildungskurses erstmals eine freiwillige schriftliche Facharztprüfung für Rheumatologie mit 34 Wahlantworten durchgeführt [2]. In den folgenden Jahren wurden für die Facharztanwärter jährlich [3], für die Mitglieder der SGR zweijährlich schriftliche Prüfungen mit 60 Wahlantwortfragen angeboten. Während für die Facharzttitelanwärter ab 2001 die Teilnahme, ab 2003 das Bestehen obligatorisch wurden, erfolgt die anonyme Teilnahme der Facharzttitelträger bis heute auf freiwilliger Basis. Diese Möglichkeit der regelmässigen Selbsteinschätzung wird von den Schweizer Rheumatologen sehr geschätzt [4].

Im Jahre 1996 fand die erste mündliche Facharztprüfung für Rheumatologie statt. Das zehnköpfige Expertenteam erarbeitete neun Fragen und erprobte den neuen Prüfungsmodus an
Oberärzten der Universitätsklinik Genf. Aufgrund der gesammelten praktischen Erfahrungen wurden die Fragen überarbeitet und anschliessend zur Prüfung der Facharztanwärter angewandt. Seither prüfen jeweils drei Teams mit je zwei Experten die Kandidaten zu neun praxisorientierten Fragen mit mehreren Teilfragen. Seit dem Jahre 2004 wird der Kandidat erst nach Bestehen der schriftlichen Prüfung zur personalaufwendigen mündlichen Prüfung zugelassen.

Als Meilenstein in der Geschichte der Schweizerischen Facharztprüfungsentwicklungen durfte die SGR im Jahre 1998 im Rahmen des Europäischen Rheumatologenkongresses EULAR in Genf 60 Wahlantwortfragen in englischer Sprache präsentieren [5]. 274 ausgefüllte Fragebogen dokumentierten den europäischen Wissensstand. Der Schwierigkeitsgrad der Fragen wurde von $2 \%$ als sehr schwierig, von $21 \%$ als schwierig und von $71 \%$ als moderat eingeschätzt. Die Korrelation der erbrachten Leistungen mit den selbstdeklarierten Fortbildungsaktivitäten ergab, dass Ärzte mit regelmässiger Teilnahme an Fortbildungsveranstaltungen mehr korrekte Antworten erzielten als Ärzte mit geringen Fortbildungsbemühungen. Aus politischen Gründen wurde auf eine Auswertung nach Nationen verzichtet.

Insgesamt wurden die Entwicklungsbemühungen honoriert durch die Evaluationskommission der FMH, welche die Examina als «vorbildlich, solid und objektiv» qualifizierte [6].

\section{Schriftliche Prüfung}

Die Prüfungskommission der SGR erarbeitete einen Blueprint, worin sowohl die beiden Dimensionen Krankheitsbild und Kategorie des ärztlichen Handelns als auch die Gewichtung der einzelnen Bereiche auf der Basis des Weiterbildungsprogramms [7] festgelegt wurden. Einerseits erfolgte die Zusammenfassung einzelner Krankheitsbilder in Krankheitsgruppen: Beispiele sind entzündliche Erkrankungen wie rheumatoide Arthritis und kristallinduzierte Arthritis, degenerative Erkrankungen wie Arthrose der peripheren und Zwischenwirbelgelenke, extraartikuläre Erkrankungen wie Epikondylitis und Schul- 
ter-Impingement-Syndrom, allgemeine Grundlagen wie pharmakotherapeutische und rehabilitative Massnahmen. Andererseits wurden wichtige ärztliche Dimensionen aufgeführt: Beispiele sind Kenntnisse der Anatomie und Pathophysiologie, Anwendung klinischer Befunderhebung und differentialdiagnostischer Überlegungen, Erstellung eines Abklärungs- oder Therapieplans. Jede einzelne Prüfungsfrage wird diesen beiden Dimensionen zugeordnet. Schliesslich wird die Gewichtung der einzelnen Teilbereiche vorgenommen und die Anzahl Fragen pro Teilbereich festgelegt. Auf diese Weise wird gewährleistet, dass das Fachgebiet der Rheumatologie dem ärztlichen Alltag entsprechend repräsentativ abgebildet wird. Als Folge der sich ändernden Abklärungs- und Therapiemöglichkeiten (z. B. Stellenwert der Sonographie am Bewegungsapparat, Einführung der TNF $\alpha$-Therapie) sowie der Überarbeitungen des Weiterbildungsprogramms passte die Prüfungskommission den Blueprint in Klausurtagungen regelmässig den neuen Begebenheiten an.

Als Format für die schriftliche Prüfung wurde die kostengünstige Form des Wahlantwortverfahrens gewählt. Beim Ausarbeiten derartiger Fragen zeigte sich bald, dass gut formulierte Lehrbuchfragen nicht immer für den rheumatologischen Alltag relevant waren. Grosses Augen- merk wird deshalb auf die Entwicklung von Vignettenfragen mit Bezug zu praktischen Patientenproblemstellungen gelegt. Beispielsweise wird bei einer klinisch relevanten Situation nach dem wichtigsten Abklärungsschritt gefragt (Beispiel 1). Bei der Generierung neuer Fragen wird zudem darauf geachtet, dass die Fragen sowohl von ambulant tätigen Ärzten der Privatpraxis wie auch von stationär tätigen Ärzten in Rehabilitations- und Universitätskliniken erstellt und überarbeitet werden.

Die statistischen Auswertungen der Fragen im Anschluss an die Prüfungen erlauben Rückschlüsse auf die Fragen. Wird eine Frage von allen Teilnehmern richtig beantwortet, so können ausgezeichnete Kandidaten nicht von weniger guten unterschieden werden. Wegen der geringen Trennschärfe macht die Aufnahme dieser Frage in den Fragenpool wenig Sinn. Wird eine Frage von der Mehrheit der Kandidaten falsch beantwortet, unabhängig von der individuellen Gesamtleistung, so muss diese eingehend erörtert werden. Oft sind kleine sprachliche Unzulänglichkeiten dafür verantwortlich, dass eine Frage nicht richtig verstanden wird. Diese Fragen werden vor der definitiven Prüfungsbewertung eliminiert (Tab. 1). Der Prozentsatz dieser für die Bewertung eliminierten Fragen steigt an, wenn viele neue und wenig bereits früher eingesetzte

Tabelle 1

Schriftliche Facharztprüfungen der Schweizerischen Gesellschaft für Rheumatologie.

\begin{tabular}{|c|c|c|c|c|c|c|c|c|c|c|}
\hline \multirow[t]{2}{*}{ Jahr } & \multicolumn{2}{|c|}{$\begin{array}{l}\text { Anzahl eliminierte } \\
\text { Fragen }\end{array}$} & \multicolumn{7}{|c|}{ Anzahl Kandidaten } & \multirow{2}{*}{\begin{tabular}{|l|}
$\begin{array}{l}\text { Anzahl } \\
\text { anonyme } \\
\text { Teilnehmer }\end{array}$ \\
$\mathbf{n}$
\end{tabular}} \\
\hline & $n$ & $\%$ & $n$ & $q$ & $\% q$ & franz. & $\%$ franz. & $\begin{array}{l}\text { erfolg- } \\
\text { reich }\end{array}$ & $\begin{array}{l}\% \text { erfolg- } \\
\text { reich }\end{array}$ & \\
\hline 1995 & 1 & 3 & 0 & 0 & 0 & 0 & 0 & 0 & 0 & 170 \\
\hline 1996 & 5 & 8 & 6 & 0 & 0 & 1 & 17 & 6 & 100 & 103 \\
\hline 1997 & 4 & 7 & 18 & 2 & 11 & 3 & 17 & 14 & 78 & 124 \\
\hline $1998^{a}$ & 5 & 8 & 10 & 4 & 40 & 2 & 20 & 7 & 70 & 274 \\
\hline 1999 & 6 & 10 & 24 & 7 & 29 & 7 & 29 & 14 & 58 & 0 \\
\hline 2000 & 4 & 7 & 14 & 4 & 29 & 3 & 21 & 9 & 64 & 188 \\
\hline $2001^{b}$ & 4 & 7 & 15 & 7 & 47 & 4 & 27 & 13 & 87 & 0 \\
\hline 2002 & 5 & 8 & 14 & 7 & 50 & 3 & 21 & 13 & 93 & 176 \\
\hline $2003^{c}$ & 2 & 3 & 10 & 5 & 50 & 2 & 20 & 10 & 100 & 0 \\
\hline 2004 & 6 & 10 & 18 & 7 & 39 & 3 & 17 & 14 & 78 & 202 \\
\hline 2005 & 2 & 2 & 14 & 5 & 36 & 2 & 14 & 14 & 100 & 0 \\
\hline Total & 44 & & 143 & 48 & & 30 & & 114 & & 1237 \\
\hline Mittelwert & 4,0 & 6,6 & 14,3 & 4,8 & 33,1 & 3,0 & 20,3 & 11,4 & 82,8 & 176,7 \\
\hline SD & 1,7 & 2,8 & 5,0 & 2,4 & 16,6 & 1,6 & 4,6 & 3,1 & 15,6 & 55,5 \\
\hline
\end{tabular}

a Prüfung anlässlich des Europäischen Rheumatologenkongresses EULAR;

b Einführung obligatorische Prüfungsteilnahme;

c Einführung obligatorisches Prüfungsbestehen; SD: Standardabweichung. 
Fragen verwendet werden. Jede einzelne bereits eingesetzte Frage ist charakterisiert durch den prozentualen Anteil richtiger Antworten sowohl bezüglich aller Teilnehmer wie bezüglich der Untergruppen der Kandidaten, Assistenzärzte in Weiterbildung, Kaderärzte an Spitälern und Ärzte in Privatpraxis.

Die Auswertungen lassen zudem Rückschlüsse auf den Schwierigkeitsgrad der Fragen zu. Der Anteil von schwierigen im Vergleich zu leichten Fragen ergibt eine Erklärung für die jährlichen Schwankungen in der Häufigkeit korrekter Antworten. Eine weitere Erklärung liegt bei der Erfahrung, dass sich einzelne Kandidaten bereits im ersten Jahr der Weiterbildung der Prüfung stellen, während die meisten, wie im Weiterbildungsprogramm vorgesehen, erst am Schluss der Weiterbildung an der Prüfung teilnehmen. In der Regel liegt der Prozentsatz der richtig beantworteten Fragen etwas über $60 \%$. Die Verteilung der erreichten Punkte fällt für verschiedene Teilnehmerkategorien unterschiedlich aus (Abb. 1). $\mathrm{Zu}$ beachten sind dabei die Tatsachen, dass alle Teilnehmer ein Interesse an Rheumatologie aufweisen und deshalb an den SGR-Fortbildungsveranstaltungen teilnehmen, dass aber nicht alle Teilnehmer an den freiwilligen, anonymen Prüfungen mit dem Ziel der Selbsteinschätzung

\section{Abbildung 1}

Leistungen an einer schriftlichen Prüfung. Geglättete Punkteverteilungen der 153 freiwilligen Teilnehmer an der anonymen Prüfung im Jahre 2002. Assistenzärzte in Weiterbildung zum Facharzt Rheumatologie; Spitalärzte tätig als Chefärzte, Leitende Ärzte oder Oberärzte mit Facharzttitel Rheumatologie oder Physikalische Medizin und Rehabilitation; Praxisärzte mit Facharzttitel Rheumatologie, Physikalische Medizin und Rehabilitation, Innere Medizin oder Allgemeinmedizin.

\section{$\%$ Teilnehmer}

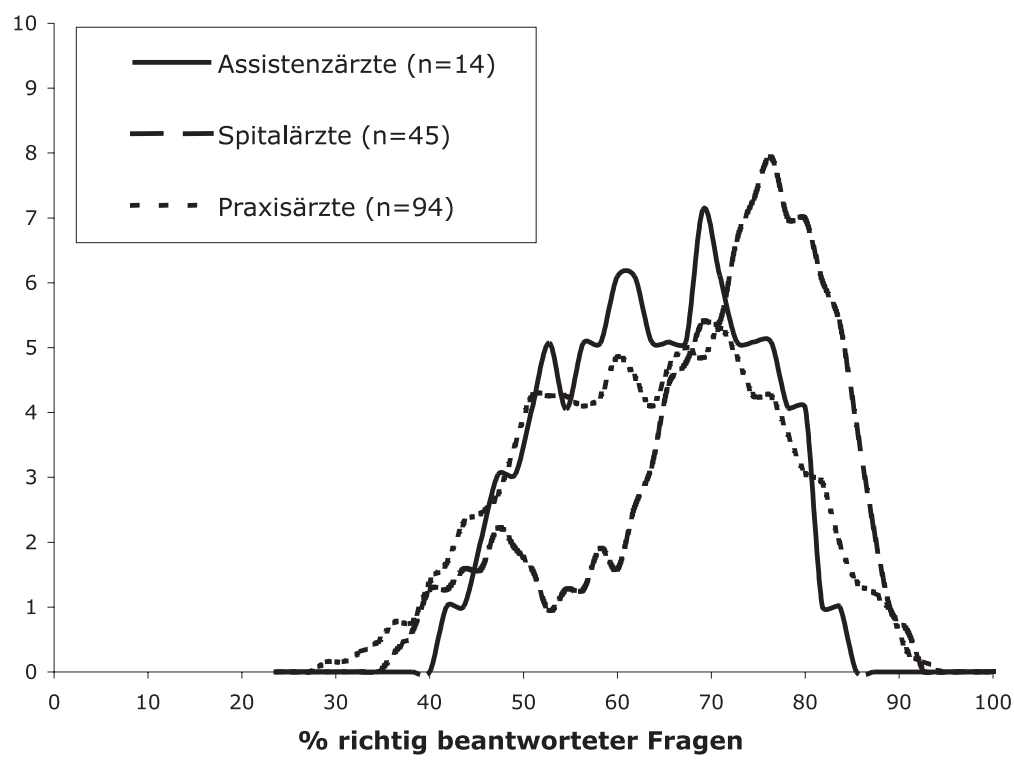

einen Facharzttitel Rheumatologie tragen oder als Rheumatologen tätig sind. Die Bestehensgrenze für die Kandidaten wird jährlich aufgrund der Erfahrungen der Vorjahre mit einzelnen identischen Fragen und, falls vorhanden, der Auswertung der Resultate der Oberärzte kalkulatorisch festgelegt.

Die Messzuverlässigkeit der Prüfungen ist zufriedenstellend, da der entsprechende Koeffizient (Cronbach alpha als Mass für die interne Konsistenz) regelmässig über dem international geforderten Standard von 0,8 für Examina von selektionierendem Charakter liegt.

\section{Mündliche Prüfung}

Die Problematik konventioneller mündlichpraktischer Prüfungen liegt in ihrer mangelhaften Messzuverlässigkeit. Nur durch eine klare Strukturierung lassen sich eine vertretbare Validität und Reliabilität erreichen. Die SGR hat sich deshalb bemüht, strukturierte Fragen zu entwickeln, welche einen Kompromiss zwischen den vorhandenen finanziellen und personellen Ressourcen und dem theoretisch optimalen Design darstellen. Die Problemstellungen gehen von einer klinischen Alltagssituation aus (kurze Fallbeschreibung, Röntgenbild, Synoviapunktat) und werden in mehreren Teilfragen erweitert analog zur Sprechstundensituation (Beispiel 2). Psychometrische Überlegungen erklären ebenso den Entscheid, auf die Gegenwart eines Patienten zu verzichten und die Untersuchungstechniken an Examinatoren vorzeigen zu lassen. Die insgesamt neun Fragen mit je durchschnittlich vier Teilfragen werden wiederum aufgrund eines Blueprints ausgewählt und umfassen die Hauptgebiete Anatomie, Pathophysiologie, Epidemiologie, Untersuchungstechniken, Labor, Bildgebung, klinische Bilder, Differentialdiagnosen, Therapiemassnahmen (medikamentös, physiotherapeutisch, rehabilitativ, chirurgisch) und soziales Netz.

Im Rahmen der Fragenentwicklung und der Schulung der zehn Examinatoren der Prüfungskommission wurde versucht, einheitliche Massstäbe zu definieren. Kernstücke sind die gemeinsame Vorgabe der korrekten Antworten und die vorgängige Festlegung der Bestehensgrenze. Bei den Teilfragen wird festgehalten, wie viele der aufgeführten Teilantworten durch die Kandidaten genannt werden müssen, damit die Teilfrage als bestanden gilt. Ebenso wird im Vorfeld entschieden, wie viele Teilfragen richtig beantwortet werden müssen, damit die Frage als bestanden gilt. Dieses Vorgehen erlaubt die vorgängige 
Gewichtung der Prüfungsinhalte. So fällt das Verpassen einer wichtigen Schlussfolgerung mehr ins Gewicht als das mangelhafte Erfassen von Detailaspekten. Reglementgemäss wird das genügende Lösen von sieben der neun Fragen gefordert, um die mündlich-praktische Facharztprüfung zu bestehen.

Damit der Einfluss der Examinatoren auf die Prüfungssituation möglichst klein ist, wird der Kandidat von insgesamt drei Teams mit je zwei Rheumatologen geprüft. Dabei kann erfasst werden, ob ein Team den Kandidaten grundsätzlich anders beurteilt als die beiden anderen Teams. Der Entscheid über das Bestehen oder Nichtbestehen der Prüfung wird bei kritischen Kandidaten im Anschluss an die Prüfung durch die unabhängig voneinander prüfenden Teams gemeinsam gefällt (Tab. 2). Dieses Vorgehen begründet sich darin, dass es bei einer kleinen Fachgesellschaft wie der SGR nicht zu vermeiden ist, dass Kandidat und Examinator bereits an derselben Klinik zusammengearbeitet haben. Erfreulicherweise konnte diese potentielle Befangenheit durch die anderen Examinatoren in den vergangenen Jahren immer aufgefangen werden.

Durch die zunehmende Strukturierung der mündlich-praktischen Prüfung hat die Messqualität zugenommen, wenn diese auch über die Jahre immer noch stark variiert. Dagegen hat die Spontaneität in den Prüfungssituationen ab- genommen. Um so wichtiger war es für die Prüfungskommission, im Sinne der Qualitätskontrolle die Prüfungen zu evaluieren. Vorerst wurden informelle Gespräche mit den Kandidaten geführt. Seit dem Jahre 2002 werden verschiedene Prüfungsaspekte standardisiert nach Expertenteam getrennt durch die Kandidaten beurteilt (Tab. 3). Erfreulicherweise wurde beispielsweise das Prüfungsklima trotz anfänglicher Bedenken von seiten der Examinatoren als durchaus positiv beurteilt.

Die Korrelation zwischen den Leistungen an der mündlich-praktischen Prüfung mit denjenigen an der schriftlichen Prüfung hat über die Jahre abgenommen (Tab. 4). Diese Beobachtung ist sicherlich durch verschiedenartige Faktoren begründet, reflektiert aber die Bemühungen, anlässlich der schrittweise weiterentwickelten mündlich-praktischen Prüfung andere Aspekte zu prüfen als durch die schriftlichen Wahlantwortfragen.

Seit dem Jahre 2001 werden zusätzlich zu den konventionellen Prüfungsinhalten weitere Kompetenzen durch die drei Expertenteams auf einer Viererskala beurteilt. Dazu gehören soziale und kommunikative Kompetenzen wie das adäquate Eingehen auf Fragen, das erläuternde Vorgehen, das konzise Vorstellen der Befunde, die überzeugende kontextbezogene Interpretation der Befunde, das verständliche Formulieren der Ant-

Tabelle 2

Mündliche Facharztprüfungen der Schweizerischen Gesellschaft für Rheumatologie.

\begin{tabular}{|c|c|c|c|c|c|c|c|}
\hline \multirow[t]{2}{*}{ Jahr } & \multicolumn{7}{|c|}{ Anzahl Kandidaten } \\
\hline & $n$ & $q$ & $\% q$ & franz. & $\%$ franz. & erfolgreich & $\begin{array}{l}\text { \% erfolg- } \\
\text { reich }\end{array}$ \\
\hline 1996 & 6 & 0 & 0 & 1 & 17 & 6 & 100 \\
\hline 1997 & 18 & 2 & 11 & 3 & 17 & 14 & 78 \\
\hline 1998 & 10 & 4 & 40 & 2 & 20 & 10 & 100 \\
\hline 1999 & 21 & 7 & 33 & 7 & 33 & 16 & 76 \\
\hline 2000 & 16 & 4 & 25 & 3 & 19 & 14 & 88 \\
\hline $2001^{a}$ & 17 & 8 & 47 & 4 & 24 & 14 & 82 \\
\hline 2002 & 13 & 6 & 46 & 3 & 23 & 11 & 85 \\
\hline $2003^{b}$ & 11 & 6 & 55 & 2 & 18 & 11 & 100 \\
\hline 2004 & 14 & 5 & 36 & 3 & 21 & 14 & 100 \\
\hline Total & 126 & 42 & & 28 & & 110 & \\
\hline Mittelwert & 14,0 & 4,7 & 32,6 & 3,1 & 21,3 & 12,2 & 89,9 \\
\hline SD & 4,6 & 2,5 & 17,8 & 1,7 & 5,0 & 3,0 & 10,2 \\
\hline
\end{tabular}

a Einführung obligatorische Prüfungsteilnahme;

b Einführung obligatorisches Prüfungsbestehen; SD: Standardabweichung. 
worten, das überzeugende Argumentieren, das situationsadäquate Verhalten. Diese Einschätzungen zeigen meistens eine signifikante Korrelation mit den Leistungen an der mündlichpraktischen, nicht aber der schriftlichen Prüfung (Tab. 4). Diese Resultate unterstreichen, dass durch die verschiedenen Prüfungsformen verschiedenartige Kompetenzen beurteilt werden und dass einzelne Kandidaten durchaus Diskrepanzen zwischen Wissen, Fertigkeiten und Verhalten zeigen können. Im Rahmen eines Pilotprojektes werden die in einer kurzen Prüfungssituation gewonnenen Einschätzungen durch die Experten verglichen mit Fremdeinschätzungen durch die Weiterbildner und Pflegenden an Weiterbildungsstätten sowie mit Selbsteinschätzungen durch die Kandidaten.

\section{Zusammenfassung und Ausblick}

Die Schweizerische Gesellschaft für Rheumatologie (SGR) blickt auf zehnjährige Erfahrungen bei den schrittweisen Entwicklungen der Facharztprüfungen zurück. Die Anfangsphase war gekennzeichnet durch die Einführung, Standardisierung und Evaluation von geeigneten schriftlichen und mündlichen Fragen. Die schriftlichen Fragen wurden nicht nur von den Kandidaten, sondern regelmässig auch von Teilnehmern an SGR-Jahrestagungen beantwortet. Dieses Vorgehen erlaubte einerseits die Überprüfung der Bestehensgrenzen der Kandidaten aufgrund der Leistungen verschiedener Berufsgruppen, andererseits die freiwilligen Selbsteinschätzungen durch die Fachärzte mit nur geringen Mehr-

Tabelle 3

Evaluationen der mündlichen Facharztprüfungen durch die Kandidaten

\begin{tabular}{|c|c|c|c|c|c|}
\hline & $\begin{array}{l}\text { Anzahl } \\
\text { Kandidaten }\end{array}$ & Prüfungsklima $^{a}$ & $\begin{array}{l}\text { Ausdrucks- } \\
\text { möglichkeiten }^{b}\end{array}$ & Zeit $^{\mathrm{c}}$ & \begin{tabular}{|l} 
Praktische \\
Relevanz $^{\text {d }}$
\end{tabular} \\
\hline 2002 & 13 & 3,85 & 3,90 & 2,33 & 3,40 \\
\hline 2003 & 11 & 3,55 & 3,64 & 3,27 & 3,64 \\
\hline 2004 & 14 & 3,83 & 3,78 & 3,07 & 4,00 \\
\hline Mittelwert & 12,7 & 3,74 & 3,77 & 2,89 & 3,68 \\
\hline SD & 1,5 & 0,17 & 0,13 & 0,50 & 0,30 \\
\hline
\end{tabular}

Mittelwerte der Antworten auf folgende Fragen:

a Prüfungsklima: 4 = angenehm; 3 = eher angenehm; 2 = eher unangenehm; 1 = unangenehm.

b Möglichkeit, Wissen und Können auszudrücken: 4 = ja; 3 = eher ja; 2 = eher nein; 1 = nein.

c Zur Verfügung stehende Zeit: 4 = zu knapp; 3 = eher zu knapp; 2 = eher zu grosszügig; 1 = zu grosszügig.

d Praktische Relevanz der Fragen: 4 = sehr relevant; 3 = relevant; 2 = wenig relevant; 1 = irrelevant.

Tabelle 4

Beziehungen zwischen den Kandidatenleistungen an den schriftlichen und mündlichen Prüfungen und den Einschätzungen der kommunikativen Kompetenzen durch die Examinatoren. Korrelationen nach Pearson, Fettdruck bei Signifikanz $p<0,05$.

\begin{tabular}{|l|l|l|l|}
\hline \multirow{5}{*}{ Schriftliche Prüfung $^{a}$} & $\begin{array}{l}\text { Kommunikative } \\
\text { Kompetenzen }^{c}\end{array}$ & ${\text { Mündliche } \text { Prüfung }^{\text {b }}}$ \\
& $2001(n=17)$ & $r=0,38(p=0,133)$ & $r=0,48(p=0,049)$ \\
\hline & $2002(n=13)$ & $r=0,46(p=0,110)$ & $r=0,58(p=0,038)$ \\
\hline & $2003(n=11)$ & $r=-0,29(p=0,387)$ & $r=0,20(p=0,556)$ \\
\hline & $2004(n=14)$ & $r=0,22(p=0,458)$ & $r=0,01(p=0,977)$ \\
\hline \multirow{5}{*}{ Mündliche Prüfung ${ }^{b}$} & $2001(n=17)$ & $r=0,88(p<0,001)$ & \\
\hline & $2002(n=13)$ & $r=0,88(p<0,001)$ & \\
\hline & $2003(n=11)$ & $r=0,65(p=0,030)$ & \\
\hline & $2004(n=14)$ & $r=0,33(p=0,249)$ & \\
\hline
\end{tabular}

a Schriftliche Prüfungen (je 60 Wahlantwortfragen);

b Mündliche Prüfung (je etwa 36 Teilfragenpunkte);

c Kommunikative Kompetenzen beurteilt durch die Examinatoren (9 Fragen). 
kosten. Dadurch können Synergien zwischen Weiterbildung und Fortbildung optimal genutzt werden. Die mündlich-praktischen Prüfungen erforderten eine klare Strukturierung. Dank vorgegebener Antwortschemata und vorgängig festgelegter Bestehensgrenzen konnten der Entscheidungsprozess transparent gestaltet, die Objektivität erhöht und die Messzuverlässigkeit verbessert werden. Während der Konsolidierungsphase wurde die Prüfungsevaluation durch die Kandidaten eingeführt. Diese beurteilten die mündlich-praktischen Prüfungen insgesamt als angenehm und attestierten ihnen eine hohe praktische Relevanz. In einer weiteren Entwicklungsphase wurden zusätzlich Einschätzungen von sozialen und kommunikativen Kandidatenkompetenzen durch die unabhängigen Expertenteams in die Prüfungen integriert. Die Validierung der Skalen wird zeigen, ob die Beurteilung weiterer Kompetenzen in den Entscheid des Bestehens oder Nichtbestehens der Facharztprüfung einfliessen und dadurch die Sicherstellung ausreichender fachärztlicher Kompetenzen breiter abstützen wird.

\section{Verdankungen}

Besonderer Dank gilt den Kandidaten, den zweijährlich Teilnehmenden der freiwilligen schriftlichen Facharztprüfung, der Prüfungskommission (Präsident: Marcel Weber; Mitglieder in elf
Jahren: André Aeschlimann, Pius Brühlmann, Christine Beyeler, Anne-Marie Chamot, Dieter Frey, Peter Grendelmeier, Pierre-André Guerne, Andreas Krebs, Beat Michel, Urs Moser, Robert Putzi, Etienne Roux, Thomas Vischer), den Koexaminatoren, den Mitarbeitern des Instituts für Medizinische Lehre der Universität Bern (insbesondere Reinhard Westkämper) sowie dem Sekretariat der Rheumaliga Schweiz (insbesondere Iris Zehnder, Susanne Frieser, Péter Tamás und Gisela Dalvit).

\section{Anhang}

\section{Beispiel 1}

\section{Beispiel einer schriftlichen Prüfungsfrage}

Ein 42jähriger Mann leidet seit 2 Tagen an einem akut schmerzhaften und geschwollenen Ellbogen. Der Patient ist adipös und hat leichtes Fieber. Sein Ellbogen ist geschwollen, überwärmt und dolent. Welches ist Ihre erste Massnahme?

A Mikroskopische Untersuchung der aspirierten Flüssigkeit.

B Bestimmung der Harnsäure im Serum.

C Blutkulturen.

D Gesamtes Blutbild.

E Bestimmung des C-reaktiven Proteins.

\section{Beispiel 2}

\section{Ausschnitt aus einer mündlichen Prüfungsfrage}

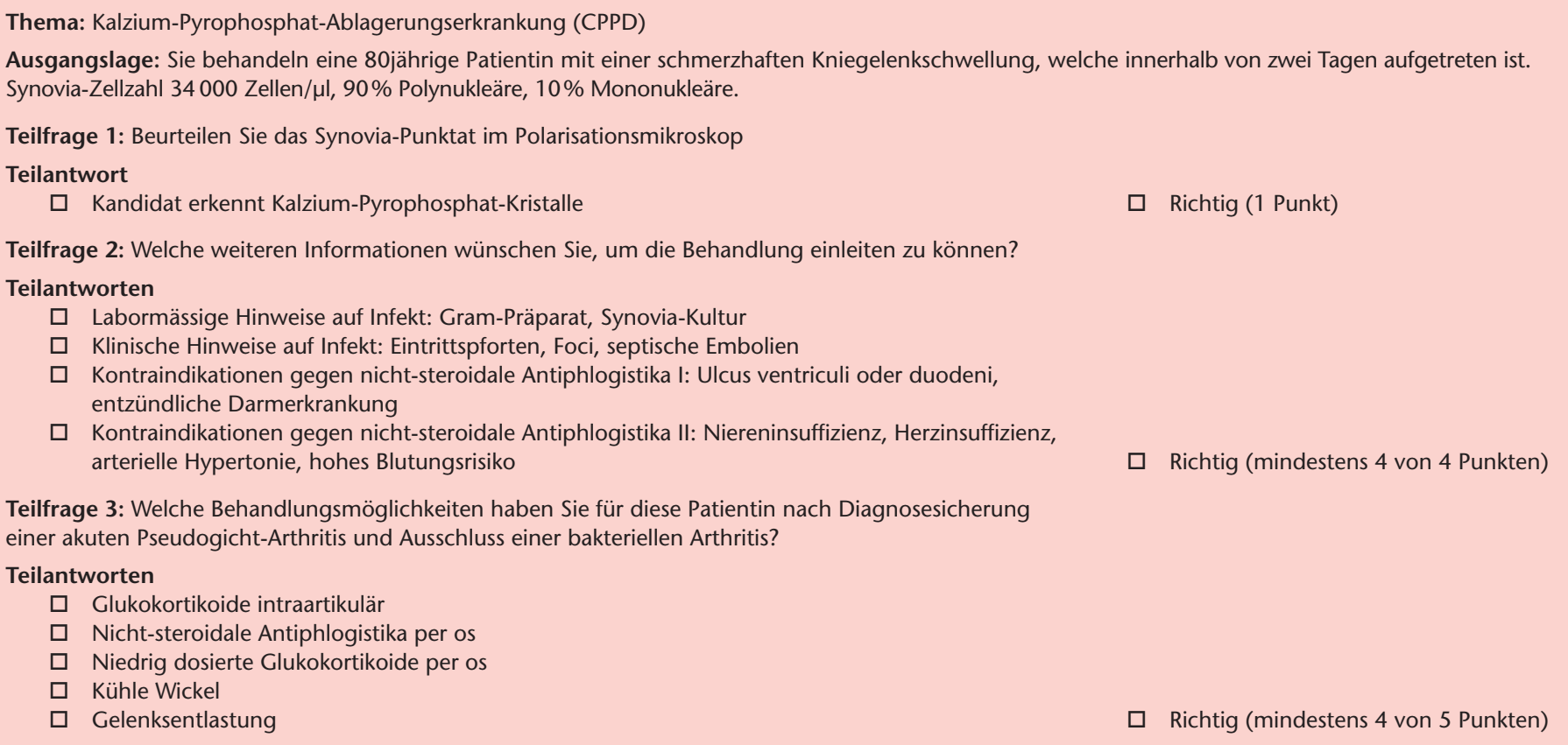

